\title{
DERIVATION OF SOLUTIONS OF THE KLEIN-GORDON EQUATION FROM SOLUTIONS OF THE WAVE EQUATION
}

\author{
by LL. G. CHAMBERS
}

(Received 2nd April 1966)

The Klein-Gordon equation

$$
\left(\nabla^{2}-\frac{\Omega^{2}}{c^{2}}-\frac{1}{c^{2}} \frac{\partial^{2}}{\partial t^{2}}\right) \psi=0
$$

$\Omega$ being a constant of dimensions $[\text { time }]^{-1}$ and $c$ being a constant velocity, appears in nuclear physics (1) and, when the Laplacian operator is twodimensional, in the theory of long gravity waves on a rotating earth (2). If $\Omega$ is zero it reduces to the wave equation

$$
\left(\nabla^{2}-\frac{1}{c^{2}} \frac{\partial^{2}}{\partial t^{2}}\right) \psi=0
$$

It does not seem to have been observed that solutions of the Klein-Gordon equation may be derived from solutions of the wave equation and it is the purpose of this note to establish that this is true.

There are three different problems which arise

(A) The solution of

$$
\left(\nabla^{2}-\frac{\Omega^{2}}{c^{2}}-\frac{1}{c^{2}} \frac{\partial^{2}}{\partial t^{2}}\right) \psi_{A}=0
$$

with $\psi=f(\boldsymbol{r}), \frac{\partial \psi}{\partial t}=0$ for $t=0$.

(B) The solution of

$$
\left(\nabla^{2}-\frac{\Omega^{2}}{c^{2}}-\frac{1}{c^{2}} \frac{\partial^{2}}{\partial t^{2}}\right) \psi_{B}=0
$$

with $\psi=0, \frac{\partial \psi}{\partial t}=g(r)$ for $t=0$.

(C) The evaluation of the Greens function

$$
G\left(r, r^{\prime} ; t, t^{\prime}\right)
$$

associated with equation (1), that is the solution of

$$
\left(\nabla^{2}-\frac{\Omega^{2}}{c^{2}}-\frac{1}{c^{2}} \frac{\partial^{2}}{\partial t^{2}}\right) G=\delta\left(r-r^{\prime}\right) \delta\left(t-t^{\prime}\right)
$$


The evaluation of $G$ enables the solution of the equation

$$
\left(\nabla^{2}-\frac{\Omega^{2}}{c^{2}}-\frac{1}{c^{2}} \frac{\dot{o}^{2}}{\partial t^{2}}\right) \psi=\chi(r, t)
$$

to be written in the form

$$
\psi(r, t)=\int d t^{\prime} \int d \tau^{\prime} G\left(r, r^{\prime} ; t, t^{\prime}\right) \chi\left(r^{\prime}, t^{\prime}\right)
$$

clearly, for the solution of (3C), it is possible to assume $r^{\prime}, t^{\prime}$ to be zero.

In the analysis which follows, the solutions of the problems with $\Omega$ zero will be given a zero subscript and the quantities $\psi_{A}, \psi_{B}, G$ will be determined in terms of $\psi_{A 0}, \psi_{B 0}, G_{0}$ respectively. This will be done by means of the Laplace Transform over time. Transforms are denoted by bars.

\section{Problem A}

Transforming equation (3A), it follows that

$$
\left(\nabla^{2}-\frac{\Omega^{2}+p^{2}}{c^{2}}\right) \Psi_{A}(r, p)+\frac{1}{c^{2}} p f(r)=0
$$

using the fact that (3), if $\phi=\phi(t)$,

$$
\int_{0}^{\infty} \frac{d^{2}}{d t^{2}}\{\phi(t)\} e^{-p t} d t=p^{2} \bar{\phi}-p \phi(0)-\phi^{\prime}(0)
$$

Similarly

$$
\left(\nabla^{2}-\frac{p^{2}}{c^{2}}\right) \bar{\psi}_{A 0}(r, p)+\frac{1}{c^{2}} p f(r)=0 .
$$

Changing $p$ to $\left(p^{2}+\Omega^{2}\right)^{\frac{1}{2}}$ in equation (7) it follows that

$$
\left(\nabla^{2}-\frac{p^{2}+\Omega^{2}}{c^{2}}\right) \Psi_{A 0}\left(r, \sqrt{p^{2}+\Omega^{2}}\right)+\frac{1}{c^{2}} \sqrt{p^{2}+\Omega^{2}} f(r)=0 .
$$

Equations (5) and (8) are consistent if

$$
\Psi_{A}(r, p)=\left(\frac{p}{p^{2}+\Omega^{2}}\right)^{\frac{1}{2}} \psi_{A 0}\left(r, \sqrt{p^{2}+\Omega^{2}}\right) .
$$

Now the inverse transform of $\bar{\psi}\left(\sqrt{p^{2}+\Omega^{2}}\right)$, dropping the spacial variation, is (4)

$$
\bar{\Psi}(t)=\psi(t)-\Omega \int_{0}^{t} \psi\left(\sqrt{t^{2}-u^{2}}\right) J_{1}(\Omega u) d u .
$$

If the inverse transform of

$$
\frac{p}{\left(p^{2}+\Omega^{2}\right)^{\frac{1}{2}}} \text { is } \lambda(t)
$$


then using the Faltung Theorem (3) the inverse transform of $\psi_{A}(r, p)$ is

$$
\int_{0}^{t} \lambda(t-v) \Psi_{A}(r, t-v) d v
$$

which is the solution of the problem. $\lambda(t)$ may be determined as follows.

We have

$$
\int_{0}^{\infty} e^{-p t} J_{0}(\Omega t)=\frac{1}{\left(p^{2}+\Omega^{2}\right)^{\frac{1}{2}}}
$$

whence

$$
\begin{aligned}
\int_{0}^{\infty} e^{-p t}\left[\frac{d}{d t} J_{0}(\Omega t)+\delta(t)\right] & \\
& =\frac{p}{\left(p^{2}+\Omega^{2}\right)^{\frac{t}{t}}}-J_{0}(0)+1 \\
& =\frac{p}{\left(p^{2}+\Omega^{2}\right)^{\frac{1}{2}}} \\
\lambda(t) & =\frac{d}{d t}\left[J_{0}(\Omega t)\right]+\delta(t) .
\end{aligned}
$$

Thus

Substituting the expression for $\lambda$ into the expression (11), it follows that

which is the required result.

$$
\psi_{A}(r, t)=\Psi_{A}(r, t)+\int_{0}^{t} \frac{d}{d v} J_{0}(\Omega v) \Psi_{A}(r, t-v) d v
$$

\section{Problem B}

Transforming equation (3B) it follows that

$$
\left(\nabla^{2}-\frac{\Omega^{2}+p^{2}}{c^{2}}\right) \Psi_{B}(r, p)+\frac{1}{c^{2}} g(r)=0
$$

on using equation (6).

Similarly

$$
\left(\nabla^{2}-\frac{p^{2}}{c^{2}}\right) \Psi_{B O}(r, p)+\frac{1}{c^{2}} g(r)=0 .
$$

Changing $p$ to $\left(p^{2}+\Omega^{2}\right)^{2}$ in equation (16) it follows that

$$
\left(\nabla^{2}-\frac{p^{2}+\Omega^{2}}{c^{2}}\right) \psi_{B 0}\left(r, \sqrt{p^{2}+\Omega^{2}}\right)+\frac{1}{c^{2}} g(r)=0 .
$$

Equations (15) and (17) are consistent if

and it follows that

$$
\bar{\psi}_{B}(r, p)=\bar{\psi}_{\mathrm{B} 0}\left(r, \sqrt{p^{2}+\Omega^{2}}\right),
$$

$$
\psi_{B}(r, t)=\psi_{B 0}(r, t)-\Omega \int_{0}^{t} \psi_{B 0}\left(r, \sqrt{t^{2}-u^{2}}\right) J_{1}(\Omega u) d u .
$$

This is the solution of problem $B$. 
It may be remarked that in the solutions of Problems A and B, $c$ need not necessarily be constant.

\section{Problem C}

The solution of

$$
\left(\nabla^{2}-\frac{1}{c^{2}} \frac{\partial^{2}}{\partial t^{2}}\right) G_{0}(r, 0 ; t ; 0)=\delta(r) \delta(t)
$$

is (6)

more generally

$$
G_{0}(r, 0 ; t, 0)=-\frac{1}{4 \pi r} \delta\left(t-\frac{r}{c}\right)
$$

$$
G_{0}\left(r, r^{\prime} ; t, t^{\prime}\right)=-\frac{1}{4 \pi R} \delta\left(t^{*}\right)
$$

where $R=\left|r-r^{\prime}\right|, t^{*}=t-t^{\prime}-\frac{R}{c}$.

Taking the Laplace transform of equation (20),

$$
\left(\nabla^{2}-\frac{p^{2}}{c^{2}}\right) \bar{G}_{0}(r, p)=\delta(r)
$$

it being assumed that the delta function is applied just after $t=0$. (If this be not the case, terms involving $\left[\frac{\partial G_{0}}{\partial t}\right]_{t=0},\left[G_{0}\right]_{t=0}$ arise.)

Similarly, the Laplace Transform of

$$
\begin{gathered}
{\left[\nabla^{2}-\frac{\Omega^{2}}{c^{2}}-\frac{1}{c^{2}} \frac{\partial^{2}}{\partial t^{2}}\right] G(\boldsymbol{r}, 0 ; t, 0)=\delta(\boldsymbol{r}) \delta(t)} \\
\left(\nabla^{2}-\frac{\Omega^{2}+p^{2}}{c^{2}}\right) \bar{G}(\boldsymbol{r}, p)=\delta(\boldsymbol{r}) .
\end{gathered}
$$

Comparing equations (23) and (25) it follows that

$$
\bar{G}(r, p)=\bar{G}_{0}\left(r, \sqrt{p^{2}+\Omega^{2}}\right) .
$$

The solution of Problem $C$ is thus similar to that of Problem B and so omitting all variables other than $t$

$$
\begin{aligned}
G(t) & =G_{0}(t)-\Omega \int_{0}^{t} G_{0}\left(\sqrt{t^{2}-u^{2}}\right) J_{1}(\Omega u) d u \\
& =G_{0}(t)-\Omega \int_{0}^{t} G_{0}(v) J_{1}\left(\Omega \sqrt{t^{2}-v^{2}}\right) \frac{v d v}{\sqrt{t^{2}-v^{2}}},
\end{aligned}
$$

on using the transformation $v^{2}=t^{2}-u^{2}$, the second form being more convenient. 
Substituting the expression (21) in equation (27), it follows that

$$
G(r, 0 ; t, 0)=-\frac{1}{4 \pi r}\left[\delta\left(t-\frac{r}{c}\right)-\frac{\Omega J_{1}\left(\Omega \sqrt{\left.t^{2}-\frac{r^{2}}{c^{2}}\right)} \frac{r}{c}\right.}{\sqrt{\left(t^{2}-\frac{r^{2}}{c^{2}}\right)}} H\left(t-\frac{r}{c}\right)\right],
$$

$H$ being the Heaviside step function. $G\left(\boldsymbol{r}, \boldsymbol{r}^{\prime} ; t, t^{\prime}\right)$ may be obtained from equation (28) by replacing $r$ by $R$ and $t$ by $t-t^{\prime}$.

The solution of equation (4) is thus given by

where

$$
\psi(\boldsymbol{r}, t)=\psi_{0}(\boldsymbol{r}, t)+\psi^{\dagger}(\boldsymbol{r}, t)
$$

$$
\psi_{0}(r, t)=-\frac{1}{4 \pi} \int \frac{\chi\left(r^{\prime}, t^{*}\right) d \tau^{\prime}}{R}
$$

the solution associated with the wave equation, and writing

$$
\begin{gathered}
\left\{\left(t-t^{\prime}\right)^{2}-\frac{R^{2}}{c^{2}}\right\}^{\frac{1}{2}}=t^{\dagger}, \\
\psi^{\dagger}(r, t)=\frac{\Omega}{4 \pi c} \int d t^{\prime} \int d \tau^{\prime} \frac{J_{1}\left(\Omega t^{\dagger}\right)}{t^{\dagger}} \chi\left(\boldsymbol{r}^{\prime}, t^{\dagger}\right)
\end{gathered}
$$

where the integral is only taken over $t^{\dagger}>0$. In effect $\psi^{\dagger}$ is the "tail " due to the existence of $\Omega$.

\section{Acknowledgment}

I am grateful to Professor I. N. Sneddon for some helpful comments on the first draft of this paper.

\section{REFERENCES}

(1) S. FlüGGE (Editor), Handbuch der Physik, Vol. 1 (Springer, Berlin, 1956), 306.

(2) J. Proudman, Dynamical Oceanography (Methuen, London, 1953), 220.

(3) W. MAGNuS and F. OBERHETTINGER, Formeln und Sätze für die Speziellen Funktionen der Mathematischen Physik (Springer, Berlin, 1948), 168.

(4) W. Magnus and F. Oberhetringer, loc. cit., 169.

(5) W. Magnus and F. Oberhettinger, loc. cit., 177.

University College of North Wales

BANGOR 